\title{
Migración infantil e inclusión educativa: Un tópico en deuda para el desarrollo de enfoques y políticas integrales de atención en Latinoamérica
}

\author{
Child Migration and Educational Inclusion, a Topic to Be Addressed for the Development of \\ Comprehensive Care Approaches and Policies in Latin America
}

\section{Migração infantil e inclusão educacional: um tema em dívida para o desenvolvimento de estratégias e políticas de atenção integral na América Latina}

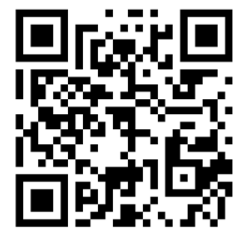

John Fredy Sánchez-Mojica Corporación Universitaria Minuto de Dios

Bogotá, Colombia

John.sanchez@uniminuto.edu.co https://orcid.org/0000-0001-7457-9885

Recibido • Received • Recebido: 03 / 02 / 2020

Corregido • Revised • Revisado: 18 / 03 / 2021

Aceptado • Accepted • Aprovado: 06 / 04 / 2021

\begin{abstract}
Resumen
Objetivo: Se plantea una reflexión s obre la forma en la c ual l os g obiernos $n$ acionales i mplementan los compromisos adquiridos para favorecer el desarrollo de la inclusión educativa para enfrentar la problemática de la migración infantil, y proponer alternativas que respondan a las verdaderas necesidades de la población afectada. Metodología. El presente documento corresponde a una revisión bibliográfica alrededor de la temática de migración infantil e inclusión educativa. Se seleccionaron un total de 29 artículos, publicados en un periodo de tiempo que va del 2004 al 2020, principalmente en la región de Latinoamérica. En conjunto, los artículos conceptualizan el tema de la inclusión educativa, analizan la problemática que se establece para la educación de calidad debido al desarrollo de las migraciones, y proponen estrategias para enfrentar dicha situación. En un primer momento del estudio se evidenció que existía una baja producción en cuanto a la temática, por lo cual se decidió evaluarla desde tres categorías diferentes: 1 . la inclusión educativa, 2. la migración y la inclusión y 3 . la migración infantil y la inclusión educativa. Dichas categorías se ubicaron de forma progresiva y permitieron delimitar un recorrido histórico sobre el desarrollo de los conceptos. Resultados. Los resultados de la investigación evidencian que el concepto de inclusión educativa aplicado a la población migrante infantil ha sido poco desarrollado. No obstante, se cuenta con reflexiones interesantes que dan cuenta de una transformación teórica, la cual debe ser plenamente explorada con la finalidad de contribuir al diseño de política pública y a transformaciones de fondo. Conclusiones. Las conclusiones sugieren que una transformación de este tipo no impacta exclusivamente a la niñez migrante, sino también a sus compañeros y compañeras de aula, sus docentes, su entorno, su familia, entre otros factores, por lo que se precisa de este proceso de reflexión para comprender la inclusión en un contexto multicultural.
\end{abstract}

Palabras clave: Inclusión educativa; educación inclusiva; migración infantil; educación de migrantes; políticas educativas. 
http://doi.org/10.15359/ree.25-2.15

http://www.una.ac.cr/educare

educare@una.ac.cr

\begin{abstract}
Objective: To reflect on how national governments implement the commitments acquired to favor the development of educational inclusion to face the problem of child migration, proposing alternatives responding to the needs of the affected population. Methodology. This paper provides a review of the literature on the theme of child migration and educational inclusion. A total of 29 articles were selected, published between 2004 and 2020, mainly in the Latin American region. All the articles conceptualize the issue of educational inclusion, analyze the problem of quality education due to the development of migration, and propose strategies to face this problem. At the beginning of the research, it is evident that there is a low production concerning this theme, so it is evaluated from three different categories: (1) educational inclusion, (2) migration and inclusion, and (3) child migration and educational inclusion. These categories were identified progressively and helped to delimit a historical overview of the development of concepts. Results. The results show that the concept of educational inclusion applied to the child migrant population has been poorly developed, regardless of interesting reflections that account for a theoretical transformation that should be explored with the proposal to contribute to the design of public policy and substantive transformations. Conclusions. The specific conclusions suggest that this transformation not only has an impact exclusively on migrant children but also on their classmates, their teachers, their environment, their family, among others, so this reflection process is required to understand inclusion in a multicultural context.
\end{abstract}

Keywords: Educational inclusion; inclusive education; child migration; migrant education; educational policies.

\title{
Resumo:
}

Objetivo. É proposta uma reflexão sobre a maneira pela qual os governos nacionais implementam os compromissos assumidos para favorecer o desenvolvimento da inclusão educacional para enfrentar o problema da migração infantil, com alternativas que respondam às verdadeiras necessidades da população afetada. Metodologia. Este documento corresponde a uma revisão de literatura sobre o tema migração infantil e inclusão educacional. Foram selecionados 29 artigos, publicados em um período de 2004 a 2020, principalmente na região da América Latina. De forma conjunta, os artigos conceituam a questão da inclusão educacional, analisam os problemas que se estabelecem para uma educação de qualidade devido ao desenvolvimento da migração e propõem estratégias para enfrentá-la. No primeiro momento da pesquisa, ficou evidente a baixa produção em relação a esse tópico, por isso foi decidido avaliá-lo em três categorias diferentes: 1. inclusão educacional, 2. migração e inclusão e 3. migração infantil e inclusão educacional. Essas categorias foram colocadas progressivamente e permitiram delimitar uma visão histórica do desenvolvimento dos conceitos. Resultados. Os resultados da pesquisa mostram que o conceito de inclusão educacional aplicado à população infantil migrante foi pouco desenvolvido. No entanto, há reflexões interessantes que explicam uma transformação teórica que deve ser totalmente explorada para contribuir com o desenho de políticas públicas e transformações fundamentais. Conclusões. As conclusões sugerem que uma transformação desse tipo não afeta exclusivamente crianças migrantes, mas também seus colegas de classe, professores, ambiente, família, entre outros, e é por isso que esse processo de reflexão é necessário para entender a inclusão em um contexto multicultural.

Palavras-chave: Inclusão educacional; educação inclusiva; migração infantil; educação de migrantes; políticas educacionais. 
http://doi.org/10.15359/ree.25-2.15

\section{Introducción}

Si bien el fenómeno de la migración ha sido frecuente a lo largo de la historia y se ha estudiado ampliamente, en particular, lo referido a las migraciones del siglo XX y sus factores asociados, en el actual siglo se presenta una complejidad del fenómeno caracterizada por una amplia variación respecto de la dirección, intensidad y composición de los flujos migratorios (Oficina Regional de la OIM para América del Sur, 2019). Esto ha obligado, tanto a la academia como a las instituciones gubernamentales, a definir una posición específica en relación con dicha problemática y a establecer políticas públicas adecuadas para garantizar un tratamiento humanitario.

La población migrante es heterogénea. Según lo demuestran los datos de United Nations Department of Economic and Social Affairs (United Nations Department of Economic and Social Affairs [UN DESA], 2019), del total de migrantes registrados para mediados de 2019, aproximadamente el $14 \%$ corresponde a personas menores de 18 años, y el $12 \%$ a personas de la tercera edad (Figura 1), lo cual significa que, en términos de composición por edad, poco más de un cuarto de la población migrante se encuentra en grupos de alta vulnerabilidad. Otros datos relevantes son que el $74 \%$ de la población migrante se encuentra en una edad laboral (entre los 20 y los 64 años), y que las mujeres representan el 48\% de esta población, mientras que los hombres llegan al 52\%.

Figura 1: Cantidad de migrantes internacionales por edad en el 2019

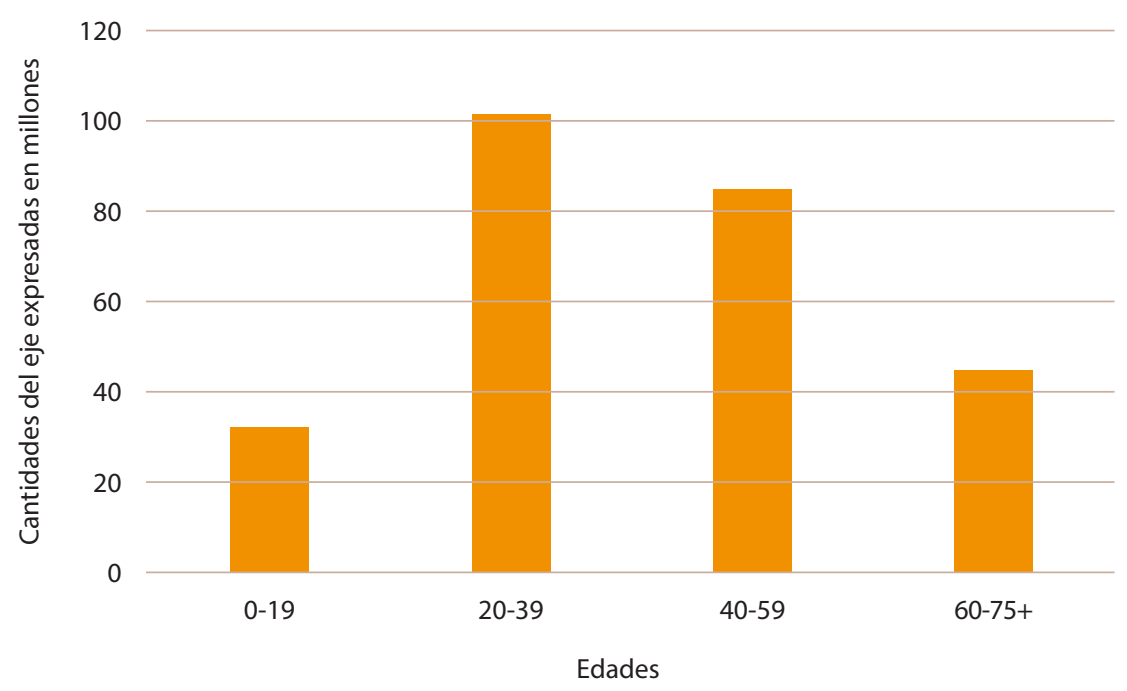

Nota: Elaboración propia con información de (UN DESA, 2019).

En particular, la población infantil migrante constituye un grupo etario de alta complejidad que requiere de un análisis profundo que dé cuenta de sus especificidades y permita diseñar políticas públicas ajustadas a sus necesidades particulares. Así como el concepto de ser 
http://doi.org/10.15359/ree.25-2.15

http://www.una.ac.cr/educare

educare@una.ac.cr

migrante difiere en función de la edad, sexo, origen, entre otros aspectos, la concepción de la infancia también depende de la condición de los niños, las niñas y adolescentes, de manera que se requiere de un enfoque diferencial para determinar las particularidades que definen a la población migrante infantil.

Ahora bien, cuando esta discusión se trae al campo de la educación, adquiere un rumbo diferente, pues se entra a discutir la necesidad de incorporar un enfoque diferencial, en tanto se trata de una población en condición de vulnerabilidad. Lo anterior obliga a poner en práctica la inclusión educativa. Este concepto, como se verá más adelante, ha tenido diferentes acepciones a lo largo de las últimas décadas; no obstante, se entiende como un modelo educativo que incorpora a todo el mundo con principios de igualdad y equidad. Por tanto, se trata del concepto adecuado para estudiar las condiciones educativas de la niñez migrante, población que posee una serie de problemáticas asociadas a su condición, la que puede ser tratada por medio de la inclusión educativa, siempre y cuando se logre caracterizar de forma adecuada.

La población migrante infantil manifiesta una problemática global, pues pone en tela de juicio los tratados que se han firmado en las últimas décadas en relación con la protección de la infancia y la adolescencia. Es importante reflexionar sobre la forma en la cual los gobiernos nacionales están poniendo en práctica los compromisos adquiridos, y proponer alternativas que respondan a las verdaderas necesidades de la población afectada.

De esta manera, un análisis que permite reconocer la manera en la cual se responde, desde el plano político, al cumplimiento de las necesidades en materia educativa de la población infantil migrante es clave para reconocer cuáles han sido los avances y las limitaciones en el desarrollo de estrategias y principios de una educación inclusiva desde la cual se comprendan las problemáticas que caracterizan, actualmente, la realidad social.

Por otro lado, a partir del análisis, es posible identificar cuáles han sido las principales barreras sociales, culturales y estructurales que existen en Latinoamérica con respecto a la educación inclusiva para la población infantil migrante; relaciona, además, las propuestas y estrategias que plantean los estudios para superar las limitaciones y establecer una mejor articulación entre el desarrollo social, la inclusión, la migración y la educación. En conjunto, el análisis planteado también permite establecer una importante contribución al campo educativo, en la medida en que se reconocen principios de acción efectivos que pueden ayudar a mejorar el acompañamiento y seguimiento, en el proceso formativo, de la población migrante en las diferentes instituciones, con base en principios de educación inclusiva.

El presente documento corresponde a una revisión bibliográfica, la cual estará guiada por tres categorías principales: la inclusión educativa, el proceso migratorio y, por último, la migración infantil y la migración inclusiva. Como se puede ver, se trata de categorías que funcionan de forma progresiva, con la finalidad de diseñar subcapítulos lineales que permitan rastrear la discusión en el tiempo. Para la búsqueda de los artículos se emplearon índices de 
búsqueda como Scopus, Scielo y Redalyc, mediante el buscador Google Académico, todo esto partiendo de los portales y repositorios de la Universidad Nacional de Costa Rica.

En total, la revisión se estableció a través de un proceso que permitió, inicialmente, seleccionar un total de 42 artículos relacionados con las variables de análisis. Luego de una lectura del resumen y de las conclusiones se escogió una muestra de 29 artículos, publicados en un periodo de tiempo que va del 2004 al 2020, principalmente en la región de Latinoamérica. Los artículos se clasificaron en cada una de las variables mencionadas, teniendo en cuenta que los objetivos y la discusión estuvieran relacionados, principalmente, con conceptualizaciones y análisis sobre la inclusión educativa, análisis sobre el proceso migratorio en Latinoamérica o problemáticas asociadas a la migración infantil. Para el anáisis de cada una de las variables, se incluyen los artículos seleccionados desde una perspectiva cronológica.

\section{Desarrollo}

Espreciso destacar que en elámbito académico se ha desarrollado ampliamentela inclusión educativa y los estudios sobre el fenómeno migratorio en el siglo XXI. No obstante, se evidencia una baja producción enfocada a la articulación de ambos conceptos, en particular en lo que refiere a la población infantil migrante. En este apartado se estudiarán, de manera particular, las dos categorías planteadas con anterioridad y se hará un rastreo de aquellas investigaciones que se acerquen a la reflexión sobre la inclusión educativa en la migración infantil.

\section{El concepto de inclusión. La inclusión educativa}

Para comprender el concepto de inclusión se requiere, necesariamente, evaluarlo en relación con la integración. La primera conceptualización de la integración se dio en el campo educativo a finales de la década de los 60, cuando las entidades de administración educativa empezaron a ser conscientes de la necesidad de incorporar, de manera efectiva, a aquel alumnado con necesidades educativas especiales (NEE). En particular, esto favoreció una composición heterogénea y diversificación dentro de los centros de enseñanza. No obstante, la innovación de dicho concepto generó, como resultado, un enfoque reduccionista que no logró aprovechar al máximo las potencialidades que evidenciaba.

De manera que, para la segunda mitad de siglo $\mathrm{XX}$, empezó a hacerse evidente la necesidad de desarrollar un enfoque que incorporara las nuevas perspectivas socioculturales y que incluyera aquellos tipos de exclusión, relacionadas con otros aspectos diferentes a las necesidades educativas especiales y a las discapacidades físicas y cognitivas del alumnado. De esta forma, surgió un segundo enfoque, el cual relaciona la inclusión con una serie de razones estructurales, asociando el tema de las condiciones socioeconómicas del estudiantado con su desempeño académico. Los pioneros en este campo serían Coleman et al. (1966), con su 
http://doi.org/10.15359/ree.25-2.15

http://www.una.ac.cr/educare

educare@una.ac.cr

reporte titulado Equality of educational opportunity, quienes analizaban los factores asociados a las desigualdades educativas en Estados Unidos y el Comité Plowden (1967) con su informe titulado Children and their primary schools que realizaba el mismo análisis en Reino Unido; cuyos resultados mostrarían que, en efecto, las condiciones socioeconómicas tienen un impacto mayor en el rendimiento que las condiciones educativas.

En particular, se puede observar cómo en el tema de la inclusión educativa se han venido estableciendo nuevos enfoques desde una perspectiva conceptual, que permiten orientar análisis más integrales sobre el tema, atendiendo a las diferentes dimensiones relacionadas con el desarrollo de las personas, y teniendo en cuenta la forma en que dichas dimensiones dependen del entorno y de las cualidades del contexto. En este sentido, es importante considerar las apreciaciones de Arnaiz Sánchez (2004), quien explica que uno de los principales problemas que presenta la educación inclusiva es que, en muchas ocasiones, las prácticas desde las cuales se trata de promover la inclusión están descontextualizadas, y se generan estrategias individualistas desde las cuales el alumnado con alguna discapacidad es el foco de toda la atención.

En este sentido, el primer elemento que se debe tener en cuenta al hablar de educación inclusiva es el de diversidad, pues permite entender y abordar un uso cada vez más amplio de la inclusión, centrada no solamente en temas de discapacidad, sino también en aspectos como la diversidad lingüística, cultural, social, de género y de acceso al conocimiento, y ligada también a una serie de factores intrapersonales que resultan ser fundamentales en el desarrollo de los procesos de aprendizaje.

También, desde una perspectiva teórica, Fernández Batanero (2005-2006) plantea que la educación inclusiva ha venido ganando terreno en el siglo XXI, en lo que tiene que ver con el rechazo a cualquier tipo de políticas desde las cuales se promueva la exclusión social. El fundamento ha sido el de responder a las necesidades de todo el alumnado desde una mirada integral, con independencia de la necesidad que presentan o de las características de tipo social o cultural que puedan generar diferencias importantes en la forma en la cual se asimilan y se comprenden los conocimientos. Por tanto, la educación inclusiva abre la posibilidad de una perspectiva en la cual estudiantes convocan y aprenden sobre diferentes condiciones sociales, capacidades e intereses, en entornos de apoyo que les permitan desarrollarse y mejorar continuamente sus procesos de formación.

Sin embargo, como lo reconoce Berruezo Adelantado (2006), si bien la educación inclusiva se ha establecido como un modelo a seguir para todas las instituciones educativas, con el fin de garantizar la atención de todo el estudiantado, sin importar sus diferencias, la realidad es que la implementación de esta práctica aún no se ha generalizado, y existen retos importantes que obligan a mejorar, principalmente, la capacitación de docentes y la articulación de los intereses y de la participación de toda la comunidad educativa. Las escuelas deben entender que la

6 
educación inclusiva es clave como mecanismo para garantizar la protección y el respeto de los Derechos Humanos, para promover una educación de calidad, y para fortalecer el desarrollo de una conciencia social. En este sentido, es preciso favorecer el análisis de los diversos componentes de tipo estructural y socioeconómico que componen la educación inclusiva.

En palabras de Moliner Miravet y Moliner García (2007), los enfoques socioeconómicos y estructurales permitían mejorar y ampliar la manera en la cual se concebía el problema de la inclusión, teniendo en cuenta que aquel: "... reduccionismo interpretativo del alumnado 'diverso' conllevaba a una atención a la diversidad centrada en la provisión de apoyos y ayudas especiales para determinados alumnos que no permite avanzar hacia una educación respetuosa con la diversidad de todos los estudiantes" (p. 2).

Con el tiempo, esta corriente se iría diversificando por medio del desarrollo de múltiples instrumentos de medición, dentro de los cuales se han categorizado las principales variables y factores socioeconómicos asociados al rendimiento del alumnado. Del mismo modo, vale la pena destacar que en los últimos años se vienen empleando índices compuestos para medir el nivel socioeconómico, lo cual ha permitido diversificar las dimensiones asociadas al bienestar de las personas. En este sentido, es importante enfocarse, a continuación, en el análisis de investigaciones en las cuales se relacionan variables socioeconómicas con el tema de la diversidad y de la inclusión en la educación.

Para empezar, cabe tener en cuenta que la mayor parte de las investigaciones que hacen parte de este segundo enfoque se limitan a establecer una correlación directa y lineal entre ambos factores, basada en la aplicación de pruebas estandarizadas que tienden a obviar otros aspectos asociados a la calidad de vida y el bienestar del estudiantado. Armenta et al. (2008) reconocen, como datos socioeconómicos relevantes, aspectos como el nivel educativo del padre y la madre, la dependencia económica, el hecho de vivir con el padre, la madre, pareja, amigos, amigas o sin nadie; el ingreso mensual, la tecnología con la que cuenta, el medio de transporte que utiliza, la situación y condiciones laborales, además de factores importantes en las relaciones interpersonales como la tenencia de una pareja, el tiempo que le dedica, si la pareja estudia o no, si trabaja o no, y si trabajan o estudian en conjunto. Si bien se trata de aspectos que otorgan datos relevantes en la práctica, es información estandarizada que deja de lado elementos con una mayor profundidad de análisis.

Es el caso de la investigación llevada a cabo por Cruz et al. (2014) en México, aplicaron la Encuesta Socioeconómica de la Asociación Mexicana de Agencias de Inteligencia de Mercado y Opinión (AMAI) y la correlacionaron con la base de datos del rendimiento académico y el patrón de alumnado becado en la institución. Por la misma línea, otras investigaciones recientes han incorporado variables y enfoques interesantes que permiten comprender la relación entre el desempeño académico y el nivel socioeconómico del estudiantado. Es el caso de la investigación llevada a cabo por González (2015), quien señala la necesidad de incorporar los factores socioeconómicos a los factores educativos. 
http://doi.org/10.15359/ree.25-2.15

http://www.una.ac.cr/educare

educare@una.ac.cr

Aspectos como la exposición a situaciones de estrés, la relación que tiene la formación con la mejora de oportunidades laborales, y demás factores contextuales y emocionales que influyen en la vida académica de los seres humanos, son factores que deben ser tomados en cuenta a la hora de plantear estrategias de mejoramiento de la educación (González, 2015).

También vale la pena destacar investigaciones recientes como la de Coschiza et. al. (2016), quienes emplearon variables de perfil general como la unidad académica a la que pertenece el alumnado, el sexo, la edad y la necesidad de traslado o desplazamiento; variables de familia núcleo como el estado civil y la cantidad de hijos e hijas y de familiares que están a su cargo; variables de perfil laboral como el tipo de ocupación, los aportes jubilatorios, las horas de trabajo y la relación del trabajo con los estudios; variables socioeconómicas (NSE) como la cobertura médica, la forma de financiamiento de los estudios y la condición de beneficiario de becas; y variables de familia de origen como la condición laboral y el nivel de estudio de los padres y madres. A partir de esto, las autoras demuestran que existe una correlación positiva entre las variables y el desempeño del alumnado, principalmente en factores como el número de hijos o hijas, la empleabilidad y la cobertura médica.

Como se puede apreciar, las investigaciones resaltan la importancia de comprender los enfoques en la educación y el desarrollo de las estrategias de un aprendizaje inclusivo a partir de observaciones integrales que permitan comprender al ser humano en cada una de sus dimensiones de desarrollo. Por ello, en este mismo sentido, resulta necesario mencionar el balance realizado por León y Collahua (2016) sobre el efecto del nivel socioeconómico en el rendimiento del estudiantado peruano en los últimos 15 años. Los autores hacen énfasis en la necesidad de diseñar instrumentos que incorporen índices compuestos:

[dado que estos] reflejan diferentes dimensiones del bienestar dentro del hogar [que] permiten contar con una medida más confiable del constructo que se quiere calcular, a diferencia de usar solo un indicador como es la educación de la madre o su estatus ocupacional [y al mismo tiempo] permite reducir los problemas de colinealidad. (Dascalu y Cozman, 2000 citadas por León y Collahua, 2016, p. 138)

Esta investigación es un referente de análisis en la región latinoamericana, pues establece un enfoque crítico sobre las tradicionales formas de medir el nivel socioeconómico y su relación el rendimiento académico. Los autores señalan que una problemática asociada a dicha medición es que en las variables que componen las dimensiones del nivel socioeconómico tienden a primar aquellas que reflejan el acceso a servicios básicos en la vivienda, así como la tenencia de activos durables y la calidad de la vivienda, no obstante, señalan León y Collahua (2016):

El acceso a servicios básicos como la electricidad, el agua y el desagüe no son necesariamente variables que permitan discriminar de manera adecuada el nivel de bienestar de las familias, puesto que solo podrían estar reflejando diferencias entre las áreas urbanas y rurales. (p. 139)

8 
Una última perspectiva es la relacionada a la inclusión de todo el alumnado según sus rasgos multiculturales, la cual tiene en cuenta todas las diferencias: de etnia, clase, género, orientación sexual, religión, entre otras. Sobre esta destaca la investigación llevada a cabo por Otálora Gallego (2017) titulada Educación inclusiva para la primera infancia en Colombia: Políticas y reformas donde la autora realiza un recorrido por las principales disposiciones en relación con la inclusión educativa en el país y concluye señalando la necesidad de emplear el concepto cultura inclusiva, considerada como una forma de ser.

Rescata el trabajo en equipo y el trabajo interdisciplinario, más allá del cumplimiento de roles y la ejecución de funciones, consolidando una sola unidad conformada por diferentes entes (padres, madres, estudiantes, docentes, orientadores, administrativos, etc.) y encaminados todos hacia la generación de un saber propio permeado por la inclusión. (Otálora Gallego, 2017, p. 26)

Se trata, por tanto, de un concepto mucho más complejo que excede el campo de las NEE así como de la interrelación rendimiento académico-NSE para entrar a convertirse en un enfoque orientado a desarrollar procesos educativos fundamentados en las múltiples dimensiones y actores que componen el trabajo pedagógico. Es necesario apuntar que este último enfoque ha sido ampliamente alimentado por la expansión de las tecnologías, lo cual ha establecido un nuevo campo de análisis que permite comprender la relación entre ambos. De esta forma, las herramientas tecnológicas se establecen como un instrumento clave que promueve el acceso de todo el estudiantado a los procesos de aprendizaje, de tal manera que pueden ser percibidas como un vehículo para superar las distintas barreras de aprendizaje a las que se enfrenta diariamente el estudiantado con algún tipo de necesidad educativa especial.

\section{El fenómeno de la migración y el enfoque inclusivo}

La inclusión social y cultural son unos de los más relevantes temas de discusión en los debates que alrededor del fenómeno migratorio se presentan. Para empezar, es necesario plantear, con Castles y Miller (2004), que la migración se puede entender como un fenómeno ligado a los procesos que han determinado la historia de la humanidad, los intercambios culturales, la estructuración de las fronteras y la transformación de los territorios. Sin embargo, de acuerdo con Levitt y Glick Schiller (2006), en la actualidad la migración se ha establecido como un concepto integral en el cual confluyen distintos tipos de motivaciones y necesidades, relacionadas con factores como la seguridad, la vulnerabilidad social y la búsqueda de nuevas oportunidades en entornos de tipo económico y productivo diferentes. Las dinámicas propias de la globalización han favorecido cada vez más la posibilidad de la migración como la búsqueda del mejoramiento de condiciones económicas o sociales con el fin de asegurar una mejor calidad de vida. 
http://doi.org/10.15359/ree.25-2.15

http://www.una.ac.cr/educare

educare@una.ac.cr

Es importante tener en cuenta que cuando las personas migran llevan consigo un conjunto de comportamientos, ideologías, costumbres y conocimientos que se ven profundamente alterados en los nuevos procesos de convivencia en un país extraño. Estas situaciones, de acuerdo con Guarnizo (2006), se intensifican mucho más cuando las razones por las cuales se genera la migración no responden a la voluntad de las personas sino a una situación de vulnerabilidad que los obliga a desplazarse de sus territorios. Lo anterior obliga a pensar que la migración no puede ser abordada únicamente a través de un análisis de tipo económico que analiza factores como las fluctuaciones de capital que se presentan, o como una competencia salarial entre personas trabajadoras locales y migrantes, sino que está compuesta por un complejo desarrollo de variables y factores de tipo social, psicológico, cultural y educativo que es preciso considerar.

Por tanto, es importante tener en cuenta, en línea con Roca i Caparà (2008), que la migración por sí misma genera una transformación social y cultural y, por tanto, contribuye a la diversificación, hibridación y multiplicación de los aspectos identitarios de los actores que intervienen en la dinámica migratoria. Sobre la relación entre ambos aspectos se ha desarrollado una producción literaria orientada principalmente en dos aspectos: a) generar un debate teórico sobre la importancia de comprender las diversas dimensiones que componen la realidad e identidad de la población migrante y b) diseñar políticas públicas de integración para migrantes.

Sobre el primero, vale la pena mencionar nuevamente a Roca i Caparà (2008), quien insiste en la necesidad de comprender los procesos de inclusión y exclusión social como factores determinantes en los procesos de socialización, enculturación y definición identitaria de la población migrante. Para el autor, los hijos e hijas de migrantes desarrollan una dinámica particularmente enriquecedora en la medida que crecen con dos referentes culturales que se encuentran en constante negociación y de los cuales surge un diálogo intercultural que contribuye, no solamente a su identidad individual, sino también a la social, en palabras de Roca i Caparà (2008): "Los hijos de personas inmigradas transfieren un plus de diversidad a nuestra sociedad. Hacen apropiación de la cultura mayoritaria, negociando imaginarios con la cultura de origen. Son productores culturales que reclaman un reconocimiento que no siempre obtienen" (p. 48)

Ahora bien, la construcción cultural y de identidad no puede ser entendida como un fenómeno individual y la población migrante no puede ser entendida como individuos que se movilizan por motivaciones personales desarticuladas entre sí. La identidad propia de los sujetos migrantes juega un papel fundamental en su proceso migratorio, llegando a desarrollar estrategias y herramientas que permitan conservarlas en el tiempo. Sobre esto, vale la pena destacar la investigación llevada a cabo por Jensen y Valdebenito (2010), quienes analizan el papel de las asociaciones de migrantes en Chile durante el proceso migratorio y su influencia en términos de inclusión. Jensen y Valdebenito (2010, citando a Fernández et al, 2002) definen el concepto de la siguiente manera: 
http://doi.org/10.15359/ree.25-2.15

La asociatividad en el contexto migratorio se constituye como un cauce de encuentro y de identificación del grupo inmigrante, reforzando los lazos internos del colectivo; según algunos autores, se constituye también como un medio idóneo para expresar su propia cultura y las necesidades de los propios inmigrantes. (p. 9)

Lo anterior supone que la necesidad de esta asociatividad surge a partir de la marcada exclusión hacia los migrantes manifestada principalmente en cuestiones semánticas, de las cuales Jensen y Valdebenito (2010) mencionan nueve: laboral, legal, habitacional, sociocultural, política, sociocomunitaria, educacional, asociativa y de salud. Por tanto, la asociatividad es pertinente en tanto permite, por un lado, identificar las semánticas de la exclusión empleadas hacia los migrantes, así como las desigualdades propias del territorio receptor $y$, por otro, generar mecanismos y espacios de empoderamiento de inmigrantes (Jensen y Valdebenito, 2010).

Dentro de esta concepción se han desarrollado enfoques diferenciales que permiten comprender el proceso identitario de acuerdo con las especificidades propias de los distintos grupos socioculturales. Ejemplo de ello es la investigación llevada a cabo por Restrepo Pineda (2017) titulada Experiencias migratorias de los varones homosexuales y bisexuales colombianos en España, en la cual el autor busca mostrar la manera como la identidad sexual influye en la motivación para iniciar y consolidar el proceso migratorio. Restrepo Pineda (2017) concluye afirmando que, aunque las motivaciones relacionadas con la identidad sexual no representan la motivación de la totalidad de la población migrante consultada, en muchas ocasiones los motivos que hicieron parte del proceso migratorio conforman una estructura integral altamente relacionada con la identidad sexual de quien migra. Este tipo de investigaciones permite comprender el fenómeno migratorio más allá de la lógica normativa que exige la compresión de la inclusión desde el enfoque clásico de integración, pues aborda la problemática en función de la construcción de identidad y no de la estandarización de las comunidades en los países de origen.

Ahora bien, sobre el segundo aspecto, relacionado con el papel de la inclusión en las políticas públicas y la población migrante, se destaca el desarrollo de la inclusión educativa como una estrategia para desarrollar procesos de articulación cultural en un espacio de alta afluencia como lo es la escuela. Es posible considerar el concepto de la inclusión educativa como un puente de comunicación entre los dos enfoques sobre los cuales se fundamenta la producción literaria alrededor de la temática. Sobre este tema se ha desarrollado una gran producción de la cual vale la pena destacar en primer lugar el trabajo realizado por Cordero Aguilar (2004) titulado Educación inclusiva. La autora parte de una definición del concepto de exclusión social para, posteriormente, realizar una caracterización de los factores que influyen en la exclusión social en América Latina, los cuales son: 
http://doi.org/10.15359/ree.25-2.15

http://www.una.ac.cr/educare

educare@una.ac.cr

- La pobreza crónica: pues la falta de capacidad para conseguir un empleo y para participar activamente en los intercambios llevados a cabo dentro de la sociedad aumentan la vulnerabilidad, "los pobres no tienen las redes sociales ni los recursos culturales para superar su pobreza lo que trae consigo la desesperación y la baja -o el comportamiento destructivo- en la juventud urbana" (Cordero Aguilar, 2004, p. 25).

- La discriminación étnica: ya que está demostrado "que las desigualdades raciales y étnicas están ligadas a la pobreza, los bajos indicadores de salud y educación, pocas oportunidades de generación de ingresos, agotamiento de los recursos naturales, migración forzada y falta de acceso al conocimiento y la información sobre sus derechos" (Cordero Aguilar, 2004, p. 25).

- Las condiciones familiares, entre ellas "el desempleo, [un] bajo nivel de educación o abusos pueden reducir seriamente las oportunidades de las futuras generaciones, creando así, un círculo vicioso de pobreza y, finalmente, la exclusión" (Cordero Aguilar, 2004, p. 26).

- Ámbito político: las deficiencias de la democracia traen como resultado la ausencia de participación en la vida política de grandes sectores sociales. El clientelismo y la corrupción, junto con la baja capacidad organizativa de muchos grupos que representan a los pobres, limitan el diálogo y la participación pública (Cordero Aguilar, 2014).

Cordero Aguilar (2004) señala que desde hace años los centros educativos han sido valorados como compensadores de las desigualdades, como instituciones capaces de asegurar la igualdad de oportunidades para todo el alumnado, garantizando su formación y progreso social. Así mismo, en una amplia investigación llevada a cabo en Costa Rica por Montero Mora (2014), el autor afirma que en este país la gestión cultural se constituye como una disciplina que, en pleno proceso de expansión, ha sido "capaz de generar un liderazgo en el desarrollo de estrategias creativas para el fomento del diálogo intercultural y del desarrollo humano" (p. 193), principalmente en lo que refiere a las estrategias de integración social de población migrante, refugiada y nacional.

Por la misma línea, desde un enfoque crítico, Ramírez Íñiguez (2016) señala nueve experiencias de inclusión educativa en Latinoamérica, y concluye que dichas propuestas se caracterizan porque responden a las necesidades específicas de cada contexto y en la definición de objetivos concretos, lo cual facilita su inserción en el ámbito institucional. Así mismo, como se mencionó con anterioridad, mediante la escuela es posible fortalecer proyectos educativos que permitan naturalizar los procesos de participación de poblaciones como la migrante, a través de la resignificación de los contenidos: "[así se estimulará] el interés y se creará un clima favorable para los aprendizajes, tanto por parte del alumnado, como por el resto de los agentes socioeducativos" (Ramírez Î́niguez, 2016, p. 187). 
También es posible rastrear un amplio contenido bibliográfico, pues se trata de investigaciones asociadas a centros o instituciones educativas de las cuales es posible extraer datos relevantes para tal propósito. Una de estas entidades es la CEPAL, la cual ha venido desarrollando una amplia producción académica sobre la temática, incluyendo el informe titulado Migración internacional e inclusión en América Latina: Análisis en los países de destino mediante encuestas de hogares (Carrasco y Suárez, 2018), en la cual se emplean como variables de análisis dos tipos de inclusión:

- Inclusión según temporalidad: estas brechas de inclusión discriminan la población local, los sujetos migrantes antiguos y los recientes, para posteriormente contrastar los perfiles de cada una en términos de sus características sociodemográficas, su inserción laboral e inclusión.

- Inclusión según procedencia:dondesediscriminan enlocales, migrantes intrarregionales y migrantes extrarregionales, las primeras corresponden a las personas que han nacido en países de América Latina y el Caribe y las segundas a los nacidos fuera de esta región.

Las anteriores investigaciones dan cuenta de la necesidad de comprender el fenómeno migratorio como una herramienta que diversifica culturalmente a la comunidad receptora y que reconoce la contribución que la población migrante hace a la local, para lo cual la educación juega un papel fundamental en tanto se establezcan las bases para una enseñanza inclusiva.

\section{Migración infantil e inclusión educativa}

Ahora bien, en lo que se refiere a la inclusión educativa dirigida a una población migrante infantil, la producción académica ha sido mucho más reducida, debido probablemente a la dificultad de trabajar con esta población en particular y también por los enfoques excesivamente amplios sobre la inclusión, como se mencionó anteriormente, que definen este concepto en función de las características socioeconómicas del individuo. Teniendo en cuenta que gran parte de las variables asociadas a la medida del nivel de inclusión educativa están orientadas a aspectos como la empleabilidad y el estado civil, se entiende que la población infantil resulte en un elemento pendiente de investigación en este campo de la investigación.

Sin embargo, vale la pena destacar algunas investigaciones recientes que han abordado la problemática tanto desde un enfoque teórico como a partir de estudios de caso. Como reflexiones conceptuales y teóricas se destacan los aportes de Vásquez (2007) en La diversidad en el aula, el gran desafío en la actualidad. ¿Están el y la docente preparados para enfrentarlo? y Migración, diversidad y el aula escolar. Tiene como propósito visualizar los diferentes enfoques que discuten sobre diversidad y multiculturalidad en la actualidad con la finalidad de trazar un camino que permita abordar la complejidad que acarrea para el docente el aumento de la migración infantil en las aulas de clase. Según Vásquez (2007), la diversidad y multiculturalidad dentro del aula debe ser vista como una variable que enriquece el contexto de esta misma, no como un obstáculo: 
http://doi.org/10.15359/ree.25-2.15

http://www.una.ac.cr/educare

educare@una.ac.cr

Nos encontramos en una época donde la importancia reside en las diferencias como referente para la construcción de una nueva escuela y una sociedad que abran las puertas a la diversidad, como cambio de pensamiento, y permiten mejorar la calidad de vida de todas las personas, independientemente de su condición. (Vázquez, 2007, p. 33)

Lo anterior significa que no es suficiente con garantizar una accesibilidad a una entidad educativa para niñez en condición de migrantes, si no se desarrolla una educación que contribuya al fortalecimiento de su identidad cultural e individual; la integración derivará en estudiantes que abandonan las aulas y pasan a ser parte de una cifra más, sin contribuir al enriquecimiento de las aulas ni a su propia formación. Vásquez (2007) hace énfasis en la necesidad de fortalecer las capacidades del personal docente que trabaja con este tipo de población, pues en la medida que este personal se encuentre capacitado y sea capaz de dejar a un lado sus prejuicios en el ejercicio pedagógico, podrá desempeñarse como un verdadero o una verdadera docente con énfasis en la inclusión.

Ahora bien, en lo referente a los estudios de caso, vale la pena destacar el realizado por Cigarroa de Aquino et al. (2016) titulado Educación inclusiva y niñez migrante: barreras a la educación de niñez de origen centroamericano en escuelas de Tapachula, Chiapas (México). En el artículo, describen tres principales barreras para desarrollar una verdadera inclusión educativa en este contexto en particular:

a) La negación en el registro para el ingreso a la escuela ...

b) El desconocimiento y la apatía en la aplicación de las leyes y reglamentos que regulan los procedimientos administrativos para el registro y egreso de niños y niñas ...

c) La indiferencia de las autoridades educativas ante situaciones de exclusión, como resultado de la discriminación fundada en prejuicios y estereotipos, mediante los cuales se considera que hijos e hijas de inmigrantes centroamericanos deben ir a escuelas de zonas marginadas de la ciudad ... (Cigarroa de Aguino et al., 2016, p. 93)

Del mismo modo, encontraron que existe una percepción negativa por parte de la niñez migrante en relación con goce efectivo de sus derechos en otro país que no es el propio. Al respecto, proponen la innovación constante para el desempeño laboral por parte del personal docente y la utilización de estrategias pedagógicas en la planificación y el abordaje de los contenidos (Cigarroa de Aquino et al., 2016); en ambos casos se requiere, necesariamente, de la articulación con las personas directivas de la institución y con los padres y las madres de familia. Esta investigación permite comprender en un contexto específico, en este caso fronterizo, la manera como opera la exclusión social y la respuesta que debe otorgar la educación inclusiva, en palabras de los autores:

La discriminación en un contexto fronterizo, como en el que se ubica Tapachula, es resultado de una construcción social que enfatiza que hay una demarcación o frontera que nos separa de "otros/as", lo que influye en una percepción negativa de estos "otros/as" 
http://doi.org/10.15359/ree.25-2.15

que nacieron del otro lado. Se asume que los derechos de una persona son "otorgados" y se ejercen en donde nació, por lo que hay quienes piensan que las personas extranjeras no tienen derechos fuera de su país de nacimiento. (Cigarroa de Aquino et al., 2016, p. 98)

En Ecuador vale la pena mencionar la investigación de Quevedo Erráez (2015) Estrategias de inclusión para estudiantes migrantes en las aulas escolares de Educación General Básica, que se trató de una recopilación y análisis de información bibliográfica realizada con la finalidad de "fundamentar la importancia del manejo de estrategias para incluir a niños y niñas inmigrantes en las aulas de clases" (Quevedo Erráez, 2015, p. 2). La investigación recoge diversas temáticas de análisis: la movilidad humana, los tipos de migración, la situación de niños, niñas y adolescentes inmigrantes en el Ecuador.

La investigadora concluye afirmando que el estudiantado migrante tiene dificultades al asistir en las instituciones educativas; no obstante, se presentan algunas estrategias para fomentar la inclusión tanto a nivel institucional como a nivel de aula que promueven la interculturalidad al tiempo que fomentan la inclusión del estudiantado inmigrante, entre ellas: el juego, el teatro, alumnado tutor, aprendizaje cooperativo, entre otras (Quevedo Erráez, 2015).

Se resaltan también las apreciaciones de Rufinelli (2017), quien explica que el personal docente debe orientar nuevas maneras de relacionarse con el estudiantado, principalmente a través del diálogo y la participación, implementando una práctica reflexiva que le permita al estudiantado convertirse en agente activo del proceso de educación. De esta forma se puede observar que las cátedras de inclusión en clase deben desarrollarse con enfoques innovadores de articulación entre padres, madres, comunidad, profesorado y docentes, de modo que los impactos de la implementación se vean reflejados a nivel social.

Así mismo, se destaca el ensayo de Cárdenas Pérez (2017) titulado "Máscaras y lenguajes" realidades en el quehacer de la inclusión educativa en Bogotá, en el cual la autora plantea una reflexión crítica en relación con las normas que promueven la inclusión educativa, pero que no garantizan la atención sistémica de la población con discapacidad. Según la autora, la inclusión educativa debe ser entendida desde un enfoque holístico que debe saber articularse con organismos, entidades, instituciones y familias de manera que puedan garantizar acciones reales que ofrezcan verdaderas soluciones y no barreras para el acceso educativo.

Por la misma línea, Poblete Melis y Galaz Valderrama (2017) en Aperturas y cierres para la inclusión educativa de niños/as migrantes en Chile plantean un estudio para determinar los factores que favorecen y las barreras que dificultan el proceso de inclusión de niñez migrante en cuatro escuelas y un liceo de Santiago. Enfatizan en la diferencia existente entre las problemáticas asociadas al sistema educativo y las que se dan a nivel interno. Según el estudio, los principales problemas son aquellos relacionados con la irregularidad migratoria de la niñez, lo cual imposibilita su entrada al sistema de educación y, por otra parte, existen profundas tensiones entre estudiantado nacional y extranjero que pueden dar pie a situaciones de bullying. 
http://doi.org/10.15359/ree.25-2.15

http://www.una.ac.cr/educare

educare@una.ac.cr

No obstante, los resultados de la investigación de Poblete Melis y Galaz Valderrama (2017) muestran que el bullying por cuestión de origen no está tan marcado, lo que se reemplaza por una indiferencia total. Otro aspecto a resaltar de esta investigación es lo que concierne a la relación entre el concepto de multiculturalidad y de inclusión, pues los autores señalan que si bien muchas instituciones se denominan como inclusivas, lo hacen porque se encuentran asociados a programas de integración para niñez con NEE y no porque se fundamenten en un enfoque realmente multicultural:

Dicho de otra manera, todos los centros educativos tienen conciencia de la presencia de niños y niñas extranjeras, pero no todos asumen en forma activa lo que significa la multiculturalidad que viven a diario. Y en ese sentido no es lo mismo que una escuela se declara inclusiva a que reconozca abiertamente la diversidad de origen de sus estudiantes como un valor. (Poblete Melisy Galaz, Valderrama, 2017, p. 256)

Otra investigación que realiza un estudio de caso es Los desafíos para la inclusión educativa de los migrantes de Estados Unidos a México (Vargas Valle, 2018). La autora inicialmente categoriza las barreras para la integración y la inclusión escolar de migrantes clasificándolas en barreras estructurales (administrativas y económicas), culturales y sociales, además, presenta una serie de acciones institucionales a favor de la integración de la niñez migrante de Estados Unidos, tal como se presenta en la Tabla 1:

Tabla 1: Estrategias para superar las barreras estructurales, culturales y sociales de integración de niñez migrante de Estados Unidos

\begin{tabular}{|c|c|c|}
\hline Tipos de barreras & Barreras & Estrategias para superarlas \\
\hline \multirow[t]{5}{*}{$\begin{array}{l}\text { Barreras } \\
\text { estructurales }\end{array}$} & Normatividad limitada & $\begin{array}{l}\text { "Difundir cambios en las normativas desde nacionales hasta } \\
\text { locales y escolares, así como entre la comunidad migrante" } \\
\text { (Vargas Valle, } 2018, \text { p. 7). }\end{array}$ \\
\hline & $\begin{array}{l}\text { Poco conocimiento sobre } \\
\text { requerimientos }\end{array}$ & $\begin{array}{l}\text { "Difundir información a nivel de las escuelas sobre los requisitos } \\
\text { para transferencias escolares" (Vargas Valle, 2018, p. 7). }\end{array}$ \\
\hline & $\begin{array}{l}\text { Ausencia de estrategias y } \\
\text { principios de acción }\end{array}$ & $\begin{array}{l}\text { “Desarrollar acciones afirmativas para dar acceso inmediato a } \\
\text { la escuela a los niños, niñas y adolescentes migrantes" (Vargas } \\
\text { Valle, } 2018, \text { p. } 7 \text { ). }\end{array}$ \\
\hline & $\begin{array}{l}\text { Falta de apoyo con las } \\
\text { familias }\end{array}$ & $\begin{array}{l}\text { "Transitar de un clima de desconfianza hacia los padres y los } \\
\text { estudiantes migrantes a otro basado en los derechos humanos" } \\
\text { (Vargas Valle, 2018, p. 7). }\end{array}$ \\
\hline & $\begin{array}{l}\text { Limitados apoyos de tipo } \\
\text { económico }\end{array}$ & $\begin{array}{l}\text { "Otorgar becas para estudiantes transnacionales de escasos } \\
\text { recursos en el periodo inmediato al arribo y posterior a éste } \\
\text { para asegurar la continuidad en la trayectoria escolar" (Vargas } \\
\text { Valle, 2018, p. 7). }\end{array}$ \\
\hline
\end{tabular}

continúa 
http://doi.org/10.15359/ree.25-2.15

\begin{tabular}{ll}
\hline $\begin{array}{l}\text { Barreras } \\
\text { culturales }\end{array}$ & $\begin{array}{l}\text { Limitada comprensión } \\
\text { sobre las capacidades del } \\
\text { alumnado migrante }\end{array}$ \\
& $\begin{array}{l}\text { Ausencia de programas } \\
\text { educativos }\end{array}$ \\
& $\begin{array}{l}\text { Limitaciones en el desarrollo } \\
\text { de estrategias inclusivas }\end{array}$
\end{tabular}

Ausencia de programas de apoyo

\section{Barreras}

sociales
Falta de capacitación

"Valorar las capacidades lingüísticas de los alumnos transnacionales; impulsar a que tengan, por ejemplo, un rol activo en las clases de inglés" (Vargas Valle, 2018, p. 7).

"Diseñar programas educativos dirigidos a población escolar que tiene el español como segundo idioma" (Vargas Valle, 2018, p. 7).

"Diseñar e implementar un manual de estrategias de educación incluyente enfocado en migrantes y dirigido a los docentes" (Vargas Valle, 2018, p. 7).

"Dar capacitación a normalistas en educación intercultural enfocada a migrantes" (Vargas Valle, 2018, p. 76).

"Disminuir la dispersión de los migrantes recién llegados. Para ello, se propone un programa piloto de escuelas ubicadas en puntos específicos donde hay mayor concentración de alumnos trasnacionales" (Vargas Valle, 2018, p. 7).

"Diseñar y poner en práctica un protocolo de bienvenida que brinde información sobre los ambientes de las escuelas en México, así como reglas que faciliten conexiones sociales con maestros y compañeros (creación de clubes, foros y reuniones), e identificar grupos vulnerables". (Vargas Valle, 2018, p. 7).

Carencia de medidas de asesoramiento

Limitaciones en la capacitación de profesionales

"Implementar un protocolo de seguimiento con asesoría psicopedagógica" (Vargas Valle, 2018, p. 7).

"Impulsar programas de capacitación para directores, orientadores y docentes en áreas de alta recepción de migrantes provenientes de Estados Unidos que acompañen la aplicación del manual y de los protocolos propuestos" (Vargas Valle, 2018, p. 7).

Problemas en la comprensión y en la comunicación

"Mejorar el nivel de inglés de profesores de esta materia" (Vargas Valle, 2018, p. 7).

"Detectar y focalizar estas acciones en los municipios y las escuelas de mayor densidad de estudiantes transnacionales $y$ de muy baja calidad" (Vargas Valle, 2018, p. 7).

Nota: Elaboración propia con base en información tomada de Vargas Valle (2018).

Como se puede apreciar, existen importantes barreras que limitan el desarrollo de la educación inclusiva para la población migrante, lo cual obliga a profundizar los análisis desde los cuales se analiza el tema de la inclusión, trascendiendo de una perspectiva reduccionista enfocada en el acceso y la cobertura, y promoviendo por otro lado una visión multidimensional en la cual se contemplen los diferentes factores personales, sociales, culturales y económicos 
http://doi.org/10.15359/ree.25-2.15

http://www.una.ac.cr/educare

educare@una.ac.cr

que se pueden aprovechar por medio de la educación y los procesos de formación, en el desarrollo de aulas de clase verdaderamente inclusivas, que valoren las percepeciones, historias de vida, pasado y proyecciones futuras de la población infantil migrante.

\section{Conclusiones}

Como se pudo observar, la producción académica en relación con la inclusión educativa ha tenido una importante transformación en las últimas décadas, abandonando el tradicional enfoque que se limitaba a la delimitación de las prioridades de la niñez y juventud con NEE (que si bien es de vital importancia para su adecuado tratamiento y acompañamiento, no proporciona una discusión a profundidad de las problemáticas asociadas a la exclusión social), para explorar alternativas mucho más diversas que permiten comprender la problemática de forma interdisciplinar. Así mismo, superar el enfoque que se limitaba al estudio de variables socioeconómicas para explicar efectos como la deserción escolar o el bajo desempeño académico supuso una comprensión mucho más compleja de la realidad sociocultural de la niñez.

Sobre la población migrante infantil, en particular, se encontró muy poca información relacionada con la atención a la diversidad. Algunas de las investigaciones consultadas muestran cómo, en efecto, el proceso migratorio transforma las construcciones identitarias y culturales tanto del sujeto migrante como del receptor, pues se realizan una serie de intercambios y negociaciones entre actores que diversifican la experiencia y mejora el conocimiento que cada parte tiene de la otra. No obstante los esfuerzos mencionados con anterioridad, en la actualidad siguen siendo frecuentes en las escuelas el rechazo y la exclusión.

El desarrollo de los procesos migratorios en el siglo XXI, en Latinoamérica, generan la necesidad de orientar una visión cada vez más profunda y multidimensional sobre la educación inclusiva, a partir del desarrollo de competencias interculturales, las cuales se establecen como una necesidad evidente en las escuelas, debido a factores como la globalización y el desarrollo de las migraciones. Estas situaciones concretas generan la necesidad de establecer estrategias que ayuden a rescatar los valores, manifestaciones y tradiciones locales; y a promover enfoques pedagógicos desde la inclusión que ayuden a poner en diálogo constante los rasgos culturales que se asocian con el desarrollo de los procesos de aprendizaje.

Sin embargo, se observa que existe una importante brecha entre lo que se dice en la teoría sobre la educación inclusiva, los acuerdos y compromisos de carácter trasnacional que orientan la implementación de medidas en las escuelas para favorecer la inclusión, y lo que sucede realmente en la práctica. Por tanto, se puede decir que, en la actualidad, los gobiernos nacionales en Latinoamérica siguen presentando una serie de retos importantes para aplicar, de manera efectiva, los compromisos adquiridos para favorecer el desarrollo de la inclusión educativa como estrategia para enfrentar las problemáticas asociadas a la migración infantil, ya 
que se siguen presentado barreras importantes que limitan la participación de los migrantes en sus espacios y entornos de aprendizaje.

Por tanto, ante este hallazgo, se puede observar la necesidad de establecer estrategias eficientes que ayuden a enfrentar la problemática de la migración infantil, proponiendo para ello alternativas que respondan a las necesidades de la población afectada. Para esto, es vital tener en cuenta la percepción de la población migrante, el conjunto de experiencias que han venido acumulando en el proceso migratorio, y las barreras que les han impedido integrarse de manera adecuada en los diferentes espacios y actividades sociales.

De acuerdo con el análisis planteado en torno al tema de la migración y los retos que se establecen para la educación inclusiva, es preciso que en las escuelas se establezcan nuevas maneras de concebir las necesidades de aprendizaje, para lo cual es preciso revisar constantemente la forma en la cual se entienden conceptos tales como la diversidad, la heterogeneidad culturas y las necesidades educativas. De esta forma, es preciso mejorar en lo que tiene que ver con el uso y distribución de los recursos humanos y materiales que existen en cada institución, para apoyar, así, el desarrollo de nuevas formas de participación.

Para finalizar, es requisito tener en cuenta que el avance teórico y metodológico mencionado tiene sus limitaciones, principalmente en lo que refiere a un enfoque diferencial para la población migrante y para la niñez. En la actualidad se considera una gama bastante amplia de variables para estudiar la problemática de la inclusión educativa. Sin embargo, en relación con la población migrante, aún no se ha desarrollado un marco de referencia considerable que permita comparar los resultados obtenidos, de manera que se trata de una discusión reciente, cuyos resultados serían de gran utilidad para el correcto tratamiento de esta población.

\section{Recomendaciones}

El análisis que se ha planteado permite reconocer la importancia para la educación de comprender el mundo simbólico de la población infantil migrante, que ayude a orientar una educación verdaderamente inclusiva que favorezca la integración, el mantenimiento y la comprensión de los elementos culturales característicos. Para ello, es preciso comprender la relación existente entre la cultura, los estilos de aprendizaje y las practicas mediante las cuales se promueve la educación inclusiva, lo cual implica reconocer la inclusión desde una dimensión totalizadora, que incluye todos aquellos elementos contextuales de los cuales depende el papel y el rol que puede desempeñar cada estudiante en sus procesos de formación y participación.

De esta manera, la inclusión educativa, en el caso de la población migrante, debe comprender, como elemento esencial, el dinamismo cultural de las sociedades, con el fin de comprender todas aquellas dimensiones que integran las experiencias de vida de la población, pues, en gran medida, sus hábitos de aprendizaje están mediados por una serie de costumbres 
http://doi.org/10.15359/ree.25-2.15

http://www.una.ac.cr/educare

educare@una.ac.cr

que dependen del contexto social del cual provienen. Así, se establece y reconoce la importancia de favorecer el desarrollo de las competencias interculturales en la educación inclusiva para la población migrante, a través del diseño y desarrollo de políticas que permitan comprender las diversas relaciones que existen entre la lengua, las experiencias, origen geográfico, identidad, conductas y comportamientos.

Es preciso favorecer el desarrollo de la competencia intercultural como un factor que le permite a las personas aplicar sus conocimientos y desenvolverse de una forma más cómoda en contextos diferentes a los de su origen. Esta competencia exige, para las comunidades académicas, el desarrollo de diferentes habilidades, destrezas y actitudes que permitan dar una respuesta adecuada a los requerimientos de tipo interpersonal generados en contextos multiculturales.

En este sentido, promover el desarrollo de la educación inclusiva implica también la necesidad de movilizar un conjunto de recursos que ayuden a potenciar las capacidades del personal docente, de las directivas, de los padres y madres de familia y de la misma población estudiantil, para reducir todas las barreas al aprendizaje y a la participación. En conjunto, un trabajo articulado enfocado en la interculturalidad ayudará a comprender mejor las perspectivas, experiencias, proyectos de vida y proyecciones de todo el alumnado, para, de esta manera, orientar mejores estrategias de formación, seguimiento y acompañamiento, que deriven en el desarrollo constante de soluciones eficientes ante el problema de la exclusión. De esta forma es posible promover las bases de una sociedad más justa y equitativa, que sabe hacer frente a las complejas dinámicas de desarrollo social en la actualidad, dentro de las cuales se destaca la migración como un elemento clave que debe ser abordado integralmente en la educación inclusiva.

\section{Referencias}

Arnaiz Sánchez, P. (2004). La educación inclusiva: Dilemas y desafíos. Revista Educación, Desarrollo y Diversidad, 7(2), 25-40.

Armenta, N. G., Pacheco, C. C. y Pineda, E. D. (2008). Factores socioeconómicos que intervienen en el desempeño académico de los estudiantes universitarios de la Facultad de Ciencias Humanas de La Universidad Autónoma de Baja California. Revista IIPSI, 11(1), 153-165. https://doi.org/10.15381/rinvp.v11i1.3888

Berruezo Adelantado, P. P. (2006). Educación inclusiva en las escuelas canadienses. Una mirada desde la perspectiva española. Revista Interuniversitaria de Formación del Profesorado, 20(2), 179-207. https://dialnet.unirioja.es/servlet/articulo?codigo=2239697

Cárdenas Pérez, G. C. (2017). "Máscaras y lenguajes" realidades en el quehacer de la inclusión educativa en Bogotá. Inclusión \& Desarrollo, 4(2), 6-10. https://doi.org/10.26620/uniminuto. inclusion.4.2.2017.6-10 
http://doi.org/10.15359/ree.25-2.15

http://www.una.ac.cr/educare educare@una.ac.cr

Castles, S., y Miller, M. J. (2004). La era de la migración. Movimientos internacionales de población en el mundo moderno. Porrúa.

Carrasco, I. y Suárez, J. I. (2018). Migración internacional e inclusión en América Latina. Análisis en los países de destino mediante encuestas de hogares. CEPAL. https://repositorio.cepal. org/handle/11362/43947

Cigarroa de Aquino, R. I., Rojas Wiesner, M. L., Evangelsita García, A. A. y Saldívar Moreno, A. (2016). Educación inclusiva y niñez migrante: Barreras a la educación de niñez de origen centroamericano en escuelas de Tapachula, Revista de Investigación en Educación, 14(1), 85-104. http://reined.webs.uvigo.es/index.php/reined/issue/view/20

Coleman, J. S., Campbell, E. Q., Hobson, C. J., McPartland, J., Mood, A. M., Weinfeld, F. D. y York, R. L. (1966). Equality of educaitonal opportunity. National Center for Educational Statistics. https://eric.ed.gov/?id=ED012275

Comité Plowden. (1967). Children and their primary schools. A report of the Central Advisory Council for Education (England) (Vol. 1:The report). HMSO. http://www.educationengland. http://www.educationengland.org.uk/documents/plowden/plowden1967-1.html

Cordero Aguilar, H. (2004). Educación inclusiva. Revista Electrónica Educare, 6, 23-38. https://doi. org/10.15359/ree.2004-6.2

Coschiza, C. C., Fernández, J. M., Gapel Redcozub, G., Nievas, M. E. y Ruiz, H. (E. 2016). Características socioeconómicas y rendimiento académico. El caso de una universidad argentina. REICE. Revista Iberoamericana sobre Calidad, Eficacia y Cambio en Educación, 14(3), 51-76. https:// doi.org/10.15366/reice2016.14.3.003

Cruz, Z., Medina, J., Vázquez, J., Espinosa, E. y Antonio, A. (2014). Influencia del nivel socioeconómico en el rendimiento académico de los alumnos del programa educativo de ingeniería industrial en la Universidad Politécnica de Altamira. En G. N. Carmona, G., Santamaría Ramírez Yuridia y L. Almanza Serrano (Coords.), Ciencias administrativas y sociales Handbook T-V: Congreso Interdisciplinario de Cuerpos Académicos (pp. 24-38). https://www.ecorfan.org/handbooks/Ciencias\%20Administrativas\%20y\%20Sociales\%20 T V/articulo 3.pdf

Fernández Batanero, J. M. (2005-2006). ¿Educaciónn inclusiva en nuestros centros educativos? Sí, pero, ¿cómo? Contextos Educativos, 8-9, 135-145. https://publicaciones.unirioja.es/ojs/ index.php/contextos/article/view/561/525

Gonzalez, E. I. (2015). Factores de contexto socioeconómicos y educativos en estudiantes de nivel superior, sugerencias para una realidad actual. Revista Interamericana de Psicologia, 49(3), 399-412. https://www.redalyc.org/articulo.oa?id=28446020010 
http://doi.org/10.15359/ree.25-2.15

http://www.una.ac.cr/educare

educare@una.ac.cr

Guarnizo, L. E. (2006). El Estado y la migración global colombiana. Migración y Desarrollo, 4(6), 79-101. https://estudiosdeldesarrollo.mx/migracionydesarrollo/numero-6/

Jensen, M. F. y Valdebenito, X. (2010). De inclusiones y exclusiones: Una perspectiva de la inmigración desde la Asociatividad en Chile. Miradas en movimiento, 3, 6-38. https:// dialnet.unirioja.es/servlet/articulo?codigo $=3182911$

Levitt, P. y Glick Schiller, L. (2006). Perspectivas internacionales sobre migración. Wellesley College y Harvard University. http://meme.phpwebhosting.com/ migracion/rimd/coleccion america latina/repensando/Repensando 7perspectivas.pdf

León, J. y Collahua, Y. (2016). El efecto del nivel socioeconómico en el rendimiento de los estudiantes peruanos: Un balance de los últimos 15 años. En grade (Ed), Investigación para el desarrollo en el Perú. Once balances (pp. 109-162). http://biblioteca.clacso.edu.ar/Peru/ grade/20170417120817/nserendimiento JL 35.pd f

Moliner Miravet, L. y Moliner García, O. (2007). Inclusión e integración: ¿Qué opinión tienen los profesionales de la educación sobre ambos conceptos. Quaderns digitals, 1-15. http:// www.quadernsdigitals.net/datos/hemeroteca/r_72/nr_770/a_10385/10385.pdf

Montero Mora, F. M. (2014). Migraciones, gestión cultural e inclusión social en Costa Rica. Periférica Internacional. Revista para el análisis de la cultura y el territorio, 15, 193-203. https://revistas. uca.es/index.php/periferica/article/view/2124/1937

Oficina Regional de la OIM para América del Sur. (2019). La migración en América delSur. Tendencias y datos relevantes. https://robuenosaires.iom.int/tendencias-y-datos-relevantes

Otálora Gallego, D. M. (2017). Educación inclusiva para la primera infancia en Colombia: Políticas y reformas. Inclusión y Desarrollo, 4(2), 21-28. https://doi.org/10.26620/uniminuto. inclusion.4.2.2017.21-28

Poblete Melis, R. y Galaz Valderrama, C. (2017). Aperturas y cierres para la inclusión educativa de niños/as migrantes en Chile. Estudios pedagógicos, 43(3), 239-257. https://doi.org/10.4067/ $\underline{\text { S0718-07052017000300014 }}$

Quevedo Erráez, J. K. (2015). Estrategias de inclusión para estudiantes migrantes en las aulas escolares de educación general básica [Tesis de licenciatura]. Universidad de Cuenca, Ecuador. http://dspace.ucuenca.edu.ec/handle/123456789/23978

Ramírez Íñiguez, A. A. (2016). Repensar la inclusión social desde la educación: Algunas experiencias en América Latina. Revista Internacional de Educación para la Justicia Social (RIEJS), 5(1), 177-194. https://revistas.uam.es/riejs/article/view/4350 
http://doi.org/10.15359/ree.25-2.15

Restrepo Pineda, J. E. (2017). Experiencias migratorias de los varones homosexuales y bisexuales colombianos en España. Revista Española de Sociología (RES), 26, 201-216. https://doi. org/10.22325/fes/res.2017.13

Roca i Caparà, N. (2008). Inmigración, identidad y procesos de inclusión y exclusión social. En La política de lo diverso: ¿Producción, reconocimiento o apropiación de lo cultural (pp. 39-50). CIDOB. https://www.cidob.org/es/media2/publicacions/monografias/la politica de lo diverso/06 roca

Rufinelli, A. (2017). Formación de docentes reflexivos: Un enfoque en construcción y disputa. Educação e Pesquisa, 43(1), 97-111. https://doi.org/10.1590/s1517-9702201701158626

United Nations Department of Economic and Social Affairs. (2019). International migrant stock 2019. https://www.un.org/en/development/desa/population/migration/data/ estimates2/estimates19.asp

Vargas Valle, E. D. (2018). Los desafíos para la inclusión educativa de los migrantes de Estados Unidos a México. CNDH, 3, 1-9. https://migracionderetorno.colmex.mx/wp-content/ uploads/2019/02/PB 3 educacion.pdf

Vásquez, E. (2007). La diversidad en el aula, el gran desafío en la actualidad.¿Está el personal docente preparado para enfrentarlo? Revista Electrónica Educare, 1, 29-46. https://doi. org/10.15359/ree.1-Ext.2 